\title{
Species sensitivity of early face and eye processing
}

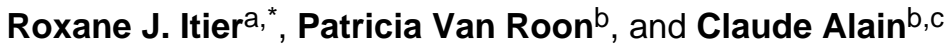 \\ aPsychology Department, University of Waterloo, Waterloo, Canada \\ ${ }^{\mathrm{b}}$ Rotman Research Institute, Baycrest Centre, Toronto, Canada \\ 'Psychology Department, University of Toronto, Toronto, Canada
}

\begin{abstract}
Humans are better at recognizing human faces than faces of other species. However, it is unclear whether this species sensitivity can be seen at early perceptual stages of face processing and whether it involves species sensitivity for important facial features like the eyes. These questions were addressed by comparing the modulations of the N170 ERP component to faces, eyes and eyeless faces of humans, apes, cats and dogs, presented upright and inverted. Although all faces and isolated eyes yielded larger responses than the control object category (houses), the N170 was shorter and smaller to human than animal faces and larger to human than animal eyes. Most importantly, while the classic inversion effect was found for human faces, animal faces yielded no inversion effect or an opposite inversion effect, as seen for objects, suggesting a different neural process involved for humans faces compared to faces of other species. Thus, in addition to its general face and eye categorical sensitivity, the N170 appears particularly sensitive to the human species for both faces and eyes. The results are discussed in the context of a recent model of the N170 response involving face and eye sensitive neurons (Itier et al., 2007) where the eyes play a central role in face perception. The data support the intuitive idea that eyes are what make animal head fronts look face-like and that proficiency for the human species involves visual expertise for the human eyes.
\end{abstract}

\section{Keywords}

Face; Eyes; Species; Perception; N170; Inversion

\section{Introduction}

The early stages of face processing have been intensively studied in the past two decades using electroencephalography techniques, with the majority of studies focusing on an early negative event-related potential (ERP) deflection, the N170, occurring between 140 and 200 $\mathrm{ms}$ after face onset over parieto-occipital scalp sites.

A host of studies have shown the strong sensitivity of N170 to human faces, as reflected by larger amplitude to faces than a variety of objects (Bentin et al., 1996; Eimer, 2000; Itier and Taylor, 2004; Itier et al., 2006; Rossion et al., 1999), whether the stimulus is a schematic, sketch, painting, drawing or photograph of a human face (Sagiv and Bentin, 2001), or a twotone Mooney figure perceived as a face (George et al., 2005; Latinus and Taylor, 2005). The N170 is generally viewed as a marker of the encoding stage of the face structure (Eimer, 2000; Rossion et al., 1999), when the perceptual representation of a face is being created,

(C) 2010 Elsevier Inc. All rights reserved.

*Corresponding author. Fax: +1 519746 8631. ritier@uwaterloo.ca (R.J. Itier). 
although its sensitivity to identity is still actively debated (e.g. Caharel et al., 2009; Heisz and Shedden, 2009; Schweinberger., in press, 2010).

Presenting faces upside-down is known to impair detection, perception and recognition of faces (Yin, 1969) due to the disruption of facial configuration (Maurer et al., 2002; Rossion and Gauthier, 2002). In comparison, perception of visual objects is less impaired by inversion because their processing is part-based (featural) rather than configural. Numerous studies have now shown that inversion also modulates the N170, with consistent increases in latency and amplitude for inverted compared to upright faces (de Haan et al., 2002; Itier and Taylor, 2002; 2004; Itier et al., 2004; Rossion et al., 2000). Importantly, this inversion effect is not found for the N170 elicited by objects (Itier et al., 2006; Rossion et al., 2000) which shows only a small latency delay and/or a small amplitude reduction (Itier et al., 2006) with this manipulation. For this reason, the N170 face inversion effect is regarded as the earliest marker of a different neuronal processing of faces compared to objects.

Although the sensitivity of the N170 to the generic face category is established, its sensitivity to the human species is unclear. Behavioral studies have shown that humans are better at recognizing human faces than the face of other species like monkeys (Pascalis et al., 1998; Pascalis et al., 2002) and this species sensitivity is established as early as 9 months of age during development (Pascalis et al., 2002). This expertise for the human face could be due to an early perceptual tuning of the visual system for the human species. The early work of Bentin et al. (1996) reported that the N170 to human faces was larger than that to animal faces (cats and owls). However, subsequent studies reported no differences between human and various animal faces when presented in natural scenes (Rousselet et al., 2004) or between human, dog and cat faces presented in close-ups (Shibata et al., 2002). Two studies reported a similar N170 amplitude for ape and human faces with longer latencies for apes (Carmel and Bentin, 2002; Itier et al., 2006) while another reported larger N170 to ape than human faces (de Haan et al., 2002). Thus, whether the human face is processed in a special way compared to other animal faces early on in the visual pathway remains unclear. In the present study we used the inversion effect to address this early species sensitivity. As mentioned above, expertise for the human face is related to configural processing which is disrupted by inversion (Maurer et al., 2002; Rossion and Gauthier, 2002). If the visual system is less tuned to the processing of animal faces, this should be due to a lack of configural processing. In contrast to the classic inversion effect for human faces, we would thus expect no inversion effect for animal faces. A lack of inversion effect has previously been reported for ape faces (de Haan et al., 2002; Itier et al., 2006) but needs to be extended to other animal species for more compelling evidence that animal and human faces are processed differently early on in the visual pathway.

Within faces, eyes bear an essential role. During the early stages of development, infants show a particular sensitivity for eyes to which they attend more than any other facial feature (Maurer, 1985). The eyes are central to all aspects of face processing including identity, emotion and gaze discrimination (see Itier and Batty, 2009 for a recent review) and the $\mathrm{N} 170$ is sensitive to eyes as shown by equally large or larger amplitudes for eyes than faces (Bentin et al., 1996; Itier et al., 2006; Itier et al., 2007; Jemel et al., 1999; Taylor et al., 2001). Because human eyes have a distinct morphology compared to eyes of other animals, including a white sclera that other species do not possess (Kobayashi and Kohshima, 1997), species sensitivity for eyes seems sensible. To our knowledge, only one previous study investigated the early neural response to eyes of various species and reported a larger N170 to human eyes compared to dog and cat eyes (Shibata et al., 2002), supporting an early species sensitivity to eyes. The eyes are at the core of a recent model of early face processing which proposes that the N170 and its modulations reflect mainly the response modulation of face and eye selective neurons (Itier et al., 2007; Itier and Batty, 2009). Given 
their central role early on in face processing, eyes could drive the response of the visual system to faces of other species. That is, the sole presence of eyes may be what defines a "face" for animal head fronts which may otherwise not be treated as faces but more as objects. Here we used eyeless faces to address this question. Previous studies have reported a delayed N170 for eyeless human faces (Eimer, 1998; Itier et al., 2007), suggesting the optimal neural response to faces requires the presence of eyes. It could thus be possible that an optimal response to the human face compared to animal faces, be also related to eyes. In other words, eyes could play a role in the species sensitivity, if it exists.

The present study sought to investigate the species sensitivity of the early face and eye processing stages as well as the role of eyes in the general response to animal faces and in this species sensitivity. We used the N170 component and its face inversion effect to assess whether human faces are processed differently from other species, early on in the visual pathway. We presented participants with upright and inverted faces, eyeless faces and isolated eye stimuli obtained from pictures of humans, apes, cats and dogs (Fig. 1), and made the following predictions. (i) If the visual system is sensitive to the species of faces, then we predict a different inversion effect for animal compared to human faces. That is, the N170 should be larger for inverted than upright human faces only. Like objects, animal faces should present no inversion effect. Alternatively, if animal faces are processed like human faces then the inversion effect should be seen for all species. (ii) Given the central role of eyes in face processing and the specific morphology of human compared to animal eyes, we predict a species sensitivity for eyes in the form of a larger N170 for human than animal eyes as reported by Shibata et al (2002). However, because eyes are features and do not yield an inversion effect (Itier et al., 2006; Itier et al., 2007), we predict no inversion effect for eyes, regardless of species. (iii) Given the central role of eyes during the early stages of face processing, we hypothesize that the general response to animal faces is largely driven by the presence of eyes which makes animal head fronts look face-like. We also hypothesize that eyes play a role in the early species sensitivity for faces, if it exists. If eyes play a similar role in the processing of other species as in the processing of human faces, then similar modulations of the N170 to the presence or absence of eyes and to isolated eyes should be seen for animals and humans. Alternatively, differences in the response to eyeless faces and isolated eyes for animals compared to humans would suggest a differential role of eyes in the processing of other species.

\section{Methods}

\section{Subjects}

Twenty-one young and healthy volunteers from the Toronto area were tested at the Rotman Research Institute and paid for their participation. Two participants were rejected due to low performances or too many artifacts. The remaining 19 participants $(20-34$ years, mean $=27.2$ years $\pm 3.6,10$ females, 15 right-handed) were included in the data analyses. All participants had normal or corrected-to-normal vision and signed a written informed consent that was approved by the Ethics Research Board of Baycrest Hospital.

\section{Stimuli and design}

Stimuli consisted of faces, eyeless faces and isolated eyes of humans, apes, cats and dogs, presented upright and inverted (48 different items per category). Forty-eight houses were also presented as a control object category, upright and upside-down. The human categories and houses were taken from Itier et al. (2007). The animal pictures were taken from various sources including the internet. Pictures of apes were restricted to great apes. Close-ups and front-views only were used for all categories. For each animal and human category, isolated eyes and eyeless faces were created from the initial whole faces using Photoshop 6.0. The 
upper and lower parts of faces were cropped so that for each picture, the eye region within the face was exactly in the center of the picture and in the center of the monitor. Faces were of neutral expression, showing all internal features and part of the hair for humans (Fig. 1). Isolated eyes were created by cropping the eye region (including eye brows) from faces in such a way that, for all stimuli, the center of the rectangular eye region was approximately the center of the eyes themselves $\left(12.3^{\circ} \times 6.4^{\circ}\right.$ visual angles $)$. Furthermore, eye stimuli were created so that all the remaining pixels of the corresponding face from which the eyes had been cut out were randomly distributed in the background. This resulted in a slightly different eye stimulus than previously used (Itier et al., 2007) but with the advantage that exactly the same pixel content was present in the full face and in the corresponding eye stimuli. Eyeless faces were created by erasing the eye region and filling it with skin-like or fur-like texture. A simple $180^{\circ}$ rotation was used to create the inverted stimuli. All pictures were presented centrally on a gray background. The eye region was centered in all stimuli (including inverted items) and on the monitor.

Participants sat in a dimly lit and sound-attenuated booth $60 \mathrm{~cm}$ from the front of a computer monitor. They performed an orientation discrimination task, pressing one button for upright and another button for inverted items, regardless of the category. Twelve blocks of 104 pictures ( 4 different pictures per category $\times 13$ categories $\times 2$ orientation) were presented twice, yielding 96 trials per category. Stimuli were on for $300 \mathrm{~ms}$ with a 1200-1500 ms randomized ISI during which a centered fixation cross appeared. Participants were instructed to respond as quickly and accurately as possible. Button keys were switched halfway through the experiment. The order of buttons, blocks, and stimuli within block were randomized and counterbalanced across participants.

\section{Electrophysiological recordings}

The Electroencephalogram (EEG) was recorded with an Active-two Biosemi system using a 66-channel elastic cap (10/20 system extended) plus five pairs of extra electrodes, for a total of 76 recording sites. Two pairs of ocular sites monitored vertical and horizontal eye movements from the outer canthi and infra-orbital ridges (IO1, IO2, LO1, LO2), one pair was situated over the mastoids (TP9/TP10), and two pairs were facial electrodes (FT9, FT10, F9, F10). The continuous EEG signal was acquired at a $516 \mathrm{~Hz}$ sampling rate with a low-pass filter of $100 \mathrm{~Hz}$. A Common Mode Sense (CMS) active electrode and Driven Right Leg (DRL) passive electrode serving as ground were used during acquisition. An average reference was computed offline and used for analyses.

Artifact correction including ocular movements was performed using Independent Component Analysis (ICA) as implemented in EEGLAB (Delorme and Makeig, 2004). ICA decomposition was derived from all trials ( -500 to $1000 \mathrm{~ms}$ epochs) concatenated across conditions. Accepted trials for which a correct response was recorded were averaged into $700 \mathrm{~ms}$ sweeps (-200 to $500 \mathrm{~ms}$ ) according to each category and orientation. Ocular artifacts were removed on each average by using ICA decomposition (Kovacevic and McIntosh, 2007). Averages were then digitally low-pass filtered $(20 \mathrm{~Hz})$.

\section{Data analysis}

The N170 peak latencies and amplitudes were measured at the maximum negativity between $130 \mathrm{~ms}$ and $210 \mathrm{~ms}$ after stimulus onset, at P7/P8, P9/P10, PO7/PO8 and CB1/CB2 electrodes. A first analysis revealed that the N170 was maximal at P9/P10 sites for all categories, therefore all subsequent analyses were conducted on these sites.

Repeated measures analyses of variance (ANOVA) were conducted separately for latency and amplitude measures, and for accuracy and reaction times (RTs). Within-subject factors 
included species (4: human, ape, cat, dog), face condition (3:eyes, face, eyeless faces) and orientation (2: upright, inverted). Hemisphere (2) was an additional factor for peak latencies and amplitudes. Houses were analyzed separately using orientation as a within-subjects factor. All ANOVAs used Greenhouse-Geisser adjusted degrees of freedom and pair-wise comparisons used Bonferroni corrections for multiple comparisons.

Because the effect of species could be confounded with low level factor differences across stimuli, we measured the mean luminance and RMS contrast of each picture using a homemade matlab program and compared species conditions using Student $t$-tests (2 tails), $p$ values corrected for multiple comparisons.

\section{Results}

\section{Luminance and RMS contrast}

Luminance and RMS contrast means for each condition are reported in Table 1. Species were compared for faces, eyes, and eyeless faces. For luminance, significant differences were found only between ape and human eyeless faces $(t(1,94)=-2.78, p<0.05)$ due to overall darker pictures for apes. For RMS contrast, differences were found between cat and human faces $(t(1,94)=-2.78, p<0.05)$, eyes $(t(1,94)=-2.85, p<0.05)$ and eyeless faces $(t(1,94)=-3.11, p<0.05)$ due to lower contrast for cats than humans. No other comparison reached significance.

\section{Behavioral analysis}

Accuracy $\left(d^{\prime}\right)$ and reaction times were each analyzed using a 4 (species) $\times 3$ (face condition) $\times 2$ (orientation) ANOVA (see Fig. 2 for means). For reaction times, a main effect of face condition $(F(2,36)=159.9, p<0.0001)$ was due to overall faster RTs for faces and eyeless faces compared to isolated eyes (Fig. 2). The main effect of species $(F(3,54)=66.37$, $p<0.0001)$ and the species $\times$ face condition interaction $(F(6,108)=8.56, p<0.0001)$ were due to systematically faster response times for the human species, but this difference was even more pronounced for eyes. Follow-up ANOVAs confirmed a main effect of species for faces $(F(3,54)=10.66, p<0.0001)$, eyeless faces $(F(3,54)=9.37, p<0.0001)$ and eyes $(F(3,54)=45.79, p<0.0001)$ with paired comparisons between human and all animal categories confirming faster RTs for humans ( $p<0.05$ or less for each comparison) while animal species did not differ significantly.

A main effect of orientation $(F(1,18)=28.07, p<0.0001)$ and an orientation $\times$ face condition interaction $(F(2,36)=23.89, p<0.0001)$ were also found, due to the orientation effect being seen for faces and eyes, with longer RTs for inverted than upright conditions but not for eyeless faces. This was confirmed by direct paired $t$-test comparisons between upright and inverted conditions for each category which were all significant $(p<0.05$ or less) except for eyeless faces of apes $(p=0.61)$, cats $(p=0.76)$, dogs $(p=0.096)$ and humans $(p=0.36)$. No orientation effect was seen for houses $(p=0.46)$.

For $d^{\prime}$ measures, the main effect of face condition $(F(2,36)=124.83, p<0.0001)$ reflected overall better performances for faces and eyeless faces compared to eyes. The main effect of species $(F(3,54)=46.8, p<0.0001)$ was significant but the species $\times$ face condition interaction $(F(6,108)=37.04, p<0.0001)$ reflected better performances for human eyes and worse performances for dog eyes while no species differences were seen for faces or eyeless faces. Follow-up ANOVAS confirmed a main effect of species for eyes $(F(3,54)=92.3, p<0.0001)$ but not for faces $(p>1)$ or eyeless faces $(p>1)$. Overall, performances decreased with inversion (main effect of orientation, $F(1,18)=19.33, p<0.0001$ ). The orientation $\times$ face condition interaction was significant $(F(2,36)=13.14, p<0.001)$ and was due to a lack of 
inversion effect for eyeless faces. Follow-up analyses confirmed a main effect of orientation for faces $(F(1,18)=13.61, p<0.005)$ and eyes $(F(1,18)=21.38, p<0.001)$ but not for eyeless faces $(p>1)$. The species $\times$ orientation interaction was also significant for eyes $(p<0.05)$ and eyeless faces $(p<0.05)$. Paired $t$-tests revealed a lack of inversion effect for human eyes $(p=0.17)$ while an inversion effect was found for animal eyes $(p<0.05)$. In contrast, an inversion effect was found for human eyeless faces $(p<0.05)$ but not for other species. Finally, there was a three-way species $\times$ orientation $\times$ face condition interaction $(F(6,108)=5.12, p<0.005)$ due to a large drop in accuracy for inverted dog eyes which were being discriminated at chance level (Fig. 2). No orientation effect was found for houses $(p=0.56)$.

\section{N170 analyses}

3.3.1. N170 peak latencies-To address the species sensitivity and its inversion effect, analyses were first conducted separately for each face condition, using a 2 (orientation) $\times$ 4 (species) $\times 2$ (hemisphere) repeated measures ANOVA.

For faces, a main effect of species $(F(3,54)=23.29, p<0.0001)$ was due to human faces yielding the shortest N170 latency ( $p<0.0001$ for each pair-wise comparison) compared to the other animal species which did not differ from each other (Fig. 3, Fig. 4). A main effect of orientation $(F(1,18)=22.27, p<.0001)$ and a species $\times$ orientation interaction $(F(3,54)=9.29$, $p<0.001$ ) were also found due to an inversion effect present for all species except for cat faces and largest for human faces (Fig. 3, Fig. 5). Follow-up ANOVAs performed on each species separately confirmed an effect of orientation for ape $(F(1,18)=10.54, p<0.005)$, dog $(F(1,18)=35.88, p<0.0001)$, human $(F(1,18)=37.26, p<0.0001)$, but not for cat faces $(p=0.95)$. When inverted faces were analyzed separately, no species effect was found, while the effect of species was present for upright faces $(F(3,54)=41.7, p<0.0001)$ analyzed separately due to shortest latencies for human faces. Thus, the shorter N170 latency for human compared to other species was seen only when faces were upright. These species differences cannot be due to mean luminance and contrast which were identical between upright and inverted pictures.

For isolated eyes, no effects were found, apart from a trend towards shorter N170s in the left compared to the right hemisphere $(F(1,18)=4.11, p=0.058)$.

Similarly, for eyeless faces, only a trend for shorter N170s in the left compared to the right hemisphere was found $(F(1,18)=4.1, p=0.058)$. No other effects were significant. However, because our previous study reported an inversion effect on the N170 latency for eyeless human faces (Itier et al., 2007), we conducted separate analyses for each species. A main effect of orientation was found for human eyeless faces $(F(1,18)=24.81, p<0.0001)$ but no orientation effect was found for ape $(p=0.81)$, cat $(p=0.76)$ or $\operatorname{dog}(p=0.99)$ eyeless faces.

To better assess the role of eyes in this species sensitivity, we also performed a 3(face type) $\times 2$ (hemisphere) ANOVA separately for each species (upright only). No effects were seen for apes and cats. For dogs, a small effect of face condition $(F(2,36)=4.28, p<0.05)$ was seen with a trend for shorter latencies for dog faces but no paired comparisons were significant. In contrast, the main effect of face condition $(F(2,36)=27.72, p<0.0001)$ was highly significant for humans, due to shorter latencies for faces than both eyes and eyeless faces ( $p$ $<0.001$ for each comparison). Eyes also yielded longer latencies than eyeless faces $(p<0.005)$. These results suggest a differential role of eyes for humans compared to other species, whether presented alone or in the context of the face. 


\section{N170 peak amplitude}

We first compared the various face conditions to the house control object category to assess the categorical sensitivity of the N170. When all upright faces were compared to upright houses, the main effect of category was highly significant $(F(4,72)=42.12, p<0.0001)$ due to houses yielding the smallest amplitudes ( $p<0.0001$ for each pair-wise comparison between houses and each species). Thus regardless of the species, the N170 was larger for faces than houses (Fig. 4). Similarly, regardless of the species, all upright eye categories yielded a larger N170 than houses $(F(4,72)=25.3, p<0.0001$, all comparisons between houses and each species at $p<0.0001)$, reflecting the general sensitivity for the N170 to isolated eyes. We also tested for the inversion effect in houses and found no significant effect of orientation $(p=0.86)$, as reported before for objects (Itier et al., 2006; Rossion et al., 2000). We also ran the analysis at occipital sites $(\mathrm{O} 1 / \mathrm{O} 2)$ where the $\mathrm{N} 170$ to houses is usually maximal and found a small inversion effect $(F(1,18)=6.42, p<0.05)$ due to smaller amplitudes for inverted compared to upright houses, i.e. an inversion effect opposite to the classic face inversion effect.

Secondly, to assess species differences, analyses were conducted separately for each face condition using a 2 (orientation) $\times 4$ (species) $\times 2$ (hemisphere) repeated measures ANOVA.

For faces, no main effects were found apart from an effect of hemisphere $(F(1,18)=11.34$, $p<0.005)$ due to larger amplitudes recorded over the right than the left hemisphere. The orientation $\times$-species $(F(3,54)=21.1, p<0.0001)$ was significant and was due to the classic inversion effect being found for human faces while no effect or an opposite inversion effect were found for the other species. Follow-up ANOVAs on each species separately confirmed the classic inversion effect for human faces $(F(1,18)=21.58, p<0.0001)$ with larger amplitudes for inverted than upright faces (Figs. 3, 5). In contrast, no effect of orientation was found for apes $(p=0.26)$. The effect of orientation was significant for cats $(F(1,18)=7.92, p<0.05)$ and $\operatorname{dogs}(F(1,18)=14.91, p<0.001)$ but was due to smaller amplitudes for inverted compared to upright cat and dog faces. In other words, an "inverted inversion effect" was found for cats and dogs. When inverted categories were analyzed separately, a main effect of species $(F(3,54)=19.6, p<0.0001)$ was due to larger amplitudes for human than other animal species (each pair-wise comparison significant at $p<0.001$ ) along with a trend for dog faces to yield smaller amplitudes than apes $(p<0.05)$. In contrast, as seen in Fig. 4, when upright faces were analyzed separately the effect of species was due to smaller amplitudes for human than animal faces $(F(3,54)=5.72, p<0.01)$, although pairwise comparisons were not significant. Thus, as predicted, the inversion effect was not present or was opposite for animal faces compared to human faces, as was seen for houses.

When eyes were analyzed separately, a main effect of species $(F(3,54)=3.05, p<0.0001)$ was due to human eyes yielding the largest amplitudes (Figs. 3, 4). Paired comparisons confirmed larger amplitudes for human than each of the other species $(p<0.005$ for each comparison) which did not differ significantly. This effect is unlikely due to low-level factors such as luminance or contrast given the only difference found was between cat and human eyes for RMS contrast, not between humans and all animal species as would be predicted if a consistent effect of luminance or contrast was driving the N170 amplitude modulations. A main effect of hemisphere was also found $(F(1,18)=5.33, p<0.05)$ due to

larger amplitudes recorded over the right than the left hemisphere. The effect of orientation was not significant nor was the orientation $\times$ species interaction $(p=0.63)$.

For eyeless faces, the effects of orientation $(F(1,18)=5.27, p<0.05)$, species $(F(3,54)=10.8$, $p<0.0001)$ and most importantly, the species $\times$ orientation interaction $(F(3,54)=17.8$, $p<0.0001)$ were all significant. Follow-up ANOVAs on each species showed no effect of orientation for apes $(p=0.78), \operatorname{dogs}(p=0.59)$, and humans $(p=0.27)$ but a main effect of 
orientation for cats $(F(1,18)=34.44, p<0.0001)$, due to smaller amplitudes for inverted than upright eyeless cat faces. When inverted categories were analyzed together, a main effect of species was found $(F(3,54)=26.27, p<0.0001)$ due to smaller amplitudes for cat and dog ( $p<0.005$ for each pair-wise comparison) compared to human and ape eyeless faces which did not differ significantly. When inverted houses were added to the analysis, the category effect was still highly significant $(p<0.0001)$ but pairwise comparisons revealed no significant difference between inverted eyeless cat faces and houses. In other words, when upside down without eyes, cat faces were no longer treated like faces but rather like objects (Figs. 3, 4). Upright eyeless face categories analyzed separately also revealed a main effect of species $(F(3,54)=3.46, p<0.05)$ due to smaller amplitudes for dogs $(p<0.05$ compared to cat eyeless faces). There was also a main effect of hemisphere $(F(1,18)=12.66, p<0.005)$ due to larger amplitudes recorded over the right than left hemisphere.

Finally, we also conducted a 3(face type) $\times 2$ (hemisphere) ANOVA separately for each species (upright only) to assess the impact of eyes. The face condition effect was significant for apes $(F(2,36)=13.38, p<0.0001)$, cats $(F(2,36)=25.88, p<0.0001)$ and dogs $(F(2,36)=41.53, p<0.0001)$, due to larger N170s for faces compared to both eyes and eyeless faces ( $p<0.001$ for each comparison). Amplitudes for eyes did not differ from that to eyeless faces for apes and cats but were larger than for eyeless faces for dogs $(p<0.05)$. In humans, the effect of face type was also significant $(F(2,36)=3.5, p<0.05)$ but paired comparisons were not; trends for smaller amplitudes for eyeless faces and larger amplitudes for eyes were seen (clear on Fig. 5). As for latencies, these results suggest a differential impact of eyes in face processing for humans compared to animals.

\section{Discussion}

Humans are face experts but this expertise seems even greater for the human species as seen by lower recognition rates for faces of other species compared to human faces (Pascalis et al., 1998; Pascalis et al., 2002). The present study investigated whether this species expertise could be related to an early perceptual tuning of the visual system for the human face. We also investigated whether the visual system was similarly species sensitive for a central facial feature, the eyes, and how the species sensitivity for faces was linked to the processing of eyes. The early perceptual stages of this species sensitivity were investigated by comparing the N170 ERP component recorded to isolated eyes, faces and eyeless faces of humans, apes, cats and dogs, presented upright and upside-down.

First, we assessed the categorical sensitivity of the N170 by comparing the neural response for upright faces and eyes of all species to houses, used here as a control object category. Regardless of the species, the N170 was indeed larger for faces and eyes than for houses, confirming its sensitivity to both face and eye categories.

Second, we focused on the species differences and on their inversion effects. We reasoned that if the visual system was less tuned to the processing of animal faces, this would involve a lack of configural processing, the hallmark of human face expertise. Given the N170 face inversion effect is due to a disruption of configural processing, we predicted a normal inversion effect for human faces but no inversion effect for animal faces. As predicted, the classic N170 increase in amplitude was found for human but not for animal faces which showed no modulation or a reduction of amplitude with inversion, like houses. In contrast, inversion delayed N170 latencies for all species, as it also does for objects (Itier et al., 2006), reflecting a general disruption of neuronal activity when visual objects are not presented in their canonical orientation. Furthermore, an earlier and smaller N170 was found for human compared to animal faces when presented upright. When presented inverted, latency differences were no longer seen while amplitudes became largest for human faces. 
These results suggest low level factors, such as contrast or luminance which were identical for upright and inverted stimuli, are unlikely driving these species differences. Rather, these modulations reflect a different neural processing of human compared to animal faces. One of these differences could be that human faces are processed faster than animal faces as reflected by shorter latencies (12-16 ms average difference between human and each species) and faster response times. We come back to the possible nature of this different neural processing of human and animal faces later in the discussion.

The reason why previous studies were inconsistent regarding the early species sensitivity of faces remains unclear. A variety of factors such as the stimuli used (e.g. natural scenes versus close-ups, animal species used), the task employed, or the population tested could have contributed to these discrepancies. Most studies also used only one animal species and did not use the inversion manipulation. The most consistent findings, however, concerns the ape species for which longer latencies (Carmel and Bentin, 2002; Itier et al., 2006) or larger amplitudes (de Haan et al., 2002) compared to human faces were reported, as found here. Two of these studies also reported no inversion effect (de Haan et al., 2002) or an inverted inversion effect (Itier et al., 2006) for ape faces. The present study replicates and extends these findings to two other animal categories, cats and dogs, hence demonstrating early species sensitivity for faces.

We also addressed the species sensitivity for the most important facial feature, the eyes. Regardless of the species, isolated eyes yielded a larger N170 response than houses, supporting the general sensitivity of the N170 to the eye category. In addition, and as predicted, no main effect of inversion or interaction between orientation and species were found, reflecting a general early sensitivity to eyes which are processed as features regardless of their orientation. However, an even larger N170 amplitude was found for human compared to animal eyes, which did not differ significantly, as also reported by one other study (Shibata et al., 2002). These results are consistent with an early species sensitivity for human eyes, as also supported by behavioral data. Indeed, participants were faster and more accurate for human than for animal eyes, regardless of their species. Furthermore, inversion did not impact the processing of human eyes while it had a detrimental effect on the processing of animal eyes (especially for dogs), reflecting a lack of expertise in accurately processing animal eyes. This particular sensitivity to human eyes is not surprising given the fundamental importance of eyes in social cognition (Emery, 2000; Itier and Batty, 2009). Furthermore, of all species, human eyes possess the largest ratio of exposed sclera size in the eye outline (Kobayashi and Kohshima, 1997). This anatomical evolution is thought to have allowed the development of an elaborated social cognition system where accurate gaze direction discrimination, even at a distance, plays a fundamental role (Emery, 2000). Moreover, human eyes are the features most attended to by infants (Maurer, 1985) who learn to orient to gaze as early as 3-4 months of age (Farroni et al., 2002; Farroni et al., 2004). It is thus not surprising that the visual system could be highly tuned to this crucially important species feature which it is used to process since birth. The current results suggest that the visual system is sensitive to this species peculiarity very early on, around $170 \mathrm{~ms}$ after stimulus onset. While mean luminance and RMS contrast contribution to this early species sensitivity for eyes was ruled out, it is very possible that other low level visual cues like eye symmetry, local contrast between iris and sclera, or configural cues of the eye region, play important roles. The precise contribution of these visual cues to the expertise for human eyes, both at the neural and behavioral level, will have to be determined by future studies.

Finally, we investigated the potential role of eyes in species sensitivity by testing the response modulation of the N170 to eyeless faces across species and by comparing the response to upright faces, eyeless faces, and eyes for each species. Although eyeless faces 
are odd looking, they are equally odd for human and animals and thus any differential effect between species is unlikely due to bizarreness alone. N170 to inverted eyeless cat faces was similar to the response to houses which suggests animal faces without eyes are sometimes treated more like objects than like faces. Normal upright animal faces, in contrast, are treated like faces because of the eyes which define a face configuration to which the visual system responds. However, as noted above, one difference between human and animal upright faces was seen in the form of earlier N170 latencies for human faces. Interestingly, this latency advantage is lost when the face is eyeless. That is, N170 latencies are no longer different between species for eyeless faces. This result suggests the presence of eyes is what produces this processing advantage for human over animal faces, an idea supported by the direct comparison of latency between faces and eyeless faces which is significant only for humans. The faster N170 for normal compared to eyeless human faces confirms previous reports (Eimer, 1998; Itier et al., 2007) and suggests the presence of eyes somehow "boosts" the processing of the face. In contrast, the N170 latency to animal faces was not influenced by the presence or absence of eyes suggesting a differential role for eyes within faces for humans compared to other species. This differential role of eyes is also seen in the face-eye comparison. For humans, eyes usually yield delayed and larger N170 than faces (Bentin et al., 1996; Itier et al., 2006; Itier et al., 2007; Jemel et al., 1999; Taylor et al., 2001), as also found in the present study (although not significant for amplitude but clearly seen on Fig. 5). In contrast, for animals, N170 was smaller for eyes than faces with no latency differences. Taken together, these results suggest that eyes contribute to a great extent in making human faces special. In other words, eyes contribute to the early species sensitivity. They are what define a face for animals yet their presence in upright faces contributes to the response differences between human and animal faces. We have tried to explain the various results of this study in the context of a recent neural model of early face processing in which the role of eyes is central (Itier et al., 2007; Itier and Batty, 2009).

This model, which is based on monkey cell recordings (Perrett et al., 1982; Perrett et al., 1985) and human intracranial data (Allison et al., 1999; McCarthy et al., 1999; Puce et al., 1999), assumes that eye and face selective neurons co-exist in the human brain and that their differential response patterns to various face stimuli can account for the N170 modulations. According to single unit recordings, face-selective cells respond to the face configuration and can also respond to isolated eyes presented outside the face context (Perrett et al., 1982, 1984, Perrett et al., 1998). In contrast, eye-selective neurons respond to isolated eyes but do not respond to the eyes of a face because of the facial configuration (Perrett et al., 1982, Perrett et al., 1985). Based on these cell properties, the model proposes that face selective neurons respond to the presentation of upright human faces while both face and eye selective neurons respond to isolated eyes, explaining the later and larger N170 to eyes compared to upright faces (Bentin et al., 1996; Itier et al., 2006; Itier et al., 2007; Jemel et al., 1999; Taylor et al., 2001). For upright faces, the configural facial context would prevent eye neurons from responding or would provoke their inhibition by face neurons (Itier and Batty, 2009). Both neuronal populations would also respond to upside-down faces, the eye neurons now responding to the eyes of the face because of the disruption of face configuration (possibly cancelling the inhibition from face neurons), yielding the classic increase in N170 amplitude and latency with face inversion. In support of their hypothesis, Itier et al. (2007) showed that inverted eyeless faces did not yield the classic N170 amplitude increase, just like other objects. In other words, taking the eyes out of the face eliminated the inversion effect because it eliminated the contribution of eye neurons to the N170 response. The N170 response to eyeless faces would also reflect the sole response of face neurons which seems optimal when eyes are present as reflected by shorter latency to faces than eyeless faces. This model, and in particular the idea that eye neurons are inhibited in response to upright but not inverted faces, was recently supported by an adaptation study (Eimer et al., 2010). 
In the context of this model, the various effects obtained for animal faces in the present study can also be explained in terms of face and eye neuron responses. The N170 to all eyes was large, although smaller to animal than human eyes. This could be due to a decreased response of eye neurons, face neurons, or both. Similarly, the absence of inversion effect for animal faces could also be due to a decreased response of eye neurons, face neurons, or both. As eye neurons do not respond to eyeless faces, this category allows monitoring the sole response of face neurons. For animals, amplitudes were smaller for eyeless than normal faces suggesting a general decreased response of face neurons to eyeless faces. The similar and sometime smaller $\mathrm{N} 170$ for inverted than upright eyeless animal faces reflects a similar or weaker response of face cells to these stimuli to the point that, for some, the response is no longer different than that to houses. Therefore, the absence of inversion effect to animal faces likely reflects a decrease in response of face neurons, rather than an absence of response of eye neurons (which respond to animal eyes) although a decrease in eye neurons response is also possible.

To conclude, the present results support the species sensitivity of early face and eye processing. While there seems to be a general response of the visual system to any face type compared to objects, this response is optimal for human faces. This species sensitivity is demonstrated by an absence of inversion effect on the N170 for animal faces, interpreted as a disruption of face neurons response to faces of other species. Similarly, a general eye processor mechanism seems to respond to any type of eyes but still shows a particular sensitivity to human eyes, possibly due to a more sensitive response of both face and eye neurons to human eyes. The eyes play a fundamental role in face perception that goes beyond that of other facial parts. In fact, the present data suggest that what make an animal's head front look like a face is mainly its eyes and that eyes play a role in the species sensitivity for human faces.

\section{Acknowledgments}

We thank Tanya Brown for help in creating the stimuli and programming and Natasa Kovacevic for help in preprocessing the data. This work was supported by funding from CIHR (MOP-89822).

\section{References}

Allison T, Puce A, Spencer DD, McCarthy G. Electrophysiological studies of human face perception. I: potentials generated in occipitotemporal cortex by face and non-face stimuli. Cereb Cortex. 1999; 9 (5):415-430. [PubMed: 10450888]

Bentin S, Allison T, Puce A, Perez E, McCarthy G. Electrophysiological studies of face perception in humans. J Cogn Neurosci. 1996; 8:551-565. [PubMed: 20740065]

Caharel S, d'Arripe O, Ramon M, Jacques C, Rossion B. Early adaptation to repeated unfamiliar faces across viewpoint changes in the right hemisphere: evidence from the N170 ERP component. Neuropsychologia. 2009; 47 (3):639-643. [PubMed: 19084547]

Carmel D, Bentin S. Domain specificity versus expertise: factors influencing distinct processing of faces. Cognition. 2002; 83:1-29. [PubMed: 11814484]

de Haan M, Pascalis O, Johnson MH. Specialization of neural mechanisms underlying face recognition in human infants. J Cogn Neurosci. 2002; 14 (2):1-11. [PubMed: 11798382]

Delorme A, Makeig S. EEGLAB: an open source toolbox for analysis of single-trial EEG dynamics including independent component analysis. J Neurosci Meth. 2004; 134 (1):9-21.

Eimer M. The face-specific N170 component reflects late stages in the structural encoding of faces. NeuroReport. 2000; 11:2319-2324. [PubMed: 10923693]

Eimer M. Does the face-specific N170 component reflect the activity of a specialized eye processor? NeuroReport. 1998; 9:2945-2948. [PubMed: 9804295]

Eimer M, Kiss M, Nicholas S. Response profile of the face-sensitive N170 component: A rapid adaptation study. Cerebral Cortex (New York, NY: 1991). 2010 
Emery NJ. The eyes have it: The neuroethology, function and evolution of social gaze. Neurosci Biobehav Rev. 2000; 24 (6):581-604. [PubMed: 10940436]

Farroni T, Massaccesi S, Pividori D, Johnson MH. Gaze following in newborns. Infancy. 2004; 5 (1): 39-60.

Farroni T, Csibra G, Simion G, Johnson MH. Eye contact detection in humans from birth. Proc Natl Acad Sci USA. 2002; 99 (14):9602-9605. [PubMed: 12082186]

George N, Jemel B, Fiori N, Chaby L, Renault B. Electrophysiological correlates of facial decision: Insights from upright and upside-down mooney-face perception. Brain Res Cogn Brain Res. 2005; 24 (3):663-673. [PubMed: 15890502]

Heisz JJ, Shedden JM. Semantic learning modifies perceptual face processing. J Cogn Neurosci. 2009; 21 (6):1127-1134. [PubMed: 18752406]

Itier RJ, Batty M. Neural bases of eye and gaze processing: the core of social cognition. Neurosci Biobehav Rev. 2009; 33 (6):843-863. [PubMed: 19428496]

Itier RJ, Taylor MJ. N170 or N1? spatiotemporal differences between object and face processing using ERPs. Cereb Cortex. 2004; 14 (2):132-142. [PubMed: 14704210]

Itier RJ, Taylor MJ. Inversion and contrast polarity reversal affect both encoding and recognition processes of unfamiliar faces: a repetition study using ERPs. Neuroimage. 2002; 15 (2):353-372. [PubMed: 11798271]

Itier RJ, Latinus M, Taylor MJ. Face, eye and object early processing: what is the face specificity? Neuroimage. 2006; 29 (2):667-676. [PubMed: 16169749]

Itier RJ, Taylor MJ, Lobaugh NJ. Spatiotemporal analysis of event-related potentials to upright, inverted, and contrast-reversed faces: effects on encoding and recognition. Psychophysiology. 2004; 41 (4):643-653. [PubMed: 15189487]

Itier RJ, Alain C, Sedore K, McIntosh AR. Early face processing specificity: it's in the eyes! J Cogn Neurosci. 2007; 19 (11):1815-1826. [PubMed: 17958484]

Jemel B, George N, Chaby L, Fiori N, Renault B. Differential processing of part-to-whole and part-topart face priming: an ERP study. NeuroReport. 1999; 10 (5):1069-1075. [PubMed: 10321486]

Kobayashi H, Kohshima S. Unique morphology of the human eye. Nature. 1997; 387 (6635):767-768. [PubMed: 9194557]

Kovacevic N, McIntosh AR. Groupwise independent component decomposition of EEG data and partial least square analysis. Neuroimage. 2007; 35 (3):1103-1112. [PubMed: 17336093]

Latinus M, Taylor MJ. Holistic processing of faces: learning effects with mooney faces. J Cogn Neurosci. 2005; 17 (8):1316-1327. [PubMed: 16197686]

Maurer, D. Infants' perception of facedness. In: Field, T.; Fox, N., editors. Social Perception in Infants. Ablex; Norwood, NJ: 1985. p. 73-100.

Maurer D, Le Grand R, Mondloch CJ. The many faces of configural processing. Trends Cogn Sci. 2002; 6 (6):255-260. [PubMed: 12039607]

McCarthy G, Puce A, Belger A, Allison T. Electrophysiological studies of human face perception. II: Response properties of face-specific potentials generated in occipitotemporal cortex. Cereb Cortex. 1999; 9 (5):431-444. [PubMed: 10450889]

Pascalis O, de Haan M, Nelson CA. Is face processing species-specific during the first year of life? Science. 2002; 296 (5571):1321-1323. [PubMed: 12016317]

Pascalis O, Coleman M, Stirnemann M, Petit O, Campbell R. Primates first? expertise affects the ability to identify individuals within and across species. Perception. 1998; 27:132b.

Perrett DI, Rolls ET, Caan W. Visual neurones responsive to faces in the monkey temporal cortex. Exp Brain Res. 1982; 47 (3):329-342. [PubMed: 7128705]

Perrett DI, Smith PA, Potter DD, Mistlin AJ, Head AS, Milner AD, Jeeves MA. Neurones responsive to faces in the temporal cortex: studies of functional organization, sensitivity to identity and relation to perception. Hum Neurobiol. 1984; 3:197-208. [PubMed: 6526706]

Perrett DI, Smith PA, Potter DD, Mistlin AJ, Head AS, Milner AD, Jeeves MA. Visual cells in the temporal cortex sensitive to face view and gaze direction. Proc R Soc Lond B Biol Sci. 1985; 223 (1232):293-317. [PubMed: 2858100] 
Perrett DI, Oram MW, Ashbridge E. Evidence accumulation in cell populations responsive to faces: an account of generalisation of recognition without mental transformations. Cognition. 1998; 67:111145. [PubMed: 9735538]

Puce A, Allison T, McCarthy G. Electrophysiological studies of human face perception. III: Effects of top-down processing on face-specific potentials. Cereb Cortex. 1999; 9 (5):445-458. [PubMed: 10450890]

Rossion B, Gauthier I. How does the brain process upright and inverted faces? Behavioural and Cognitive Neuroscience Reviews. 2002; 1 (1):62-74.

Rossion B, Gauthier I, Tarr MJ, Despland P, Bruyer R, Linotte S, Crommelinck M. The N170 occipito-temporal component is delayed and enhanced to inverted faces but not to inverted objects: an electrophysiological account of face-specific processes in the human brain. NeuroReport. 2000; 11 (120147456):69-74. [PubMed: 10683832]

Rossion B, Campanella S, Gomez CM, Delinte A, Debatisse D, Liard L, Dubois S, Bruyer R, Crommelinck M, Guerit J. Task modulation of brain activity related to familiar and unfamiliar face processing: an ERP study. Clin Neurophysiol. 1999:449-462. [PubMed: 10363769]

Rousselet GA, Mace MJ, Fabre-Thorpe M. Animal and human faces in natural scenes: how specific to human faces is the N170 ERP component? J Vis. 2004; 4 (1):13-21. [PubMed: 14995895]

Sagiv N, Bentin S. Structural encoding of human and schematic faces: holistic and part-based processes. J Cogn Neurosci. 2001; 13:937-951. [PubMed: 11595097]

Schweinberger, SR. Neurophysiological correlates of face recognition. In: Calder, AJ.; Rhodes, G.; Johnson, MH.; Haxby, JV., editors. Handbook of face perception. Oxford University Press; Oxford: 2010. in press

Shibata T, Nishijo H, Tamura R, Miyamoto K, Eifuku S, Endo S, Ono T. Generators of visual evoked potentials for faces and eyes in the human brain as determined by dipole localization. Brain Topogr. 2002; 15 (1):51-63. [PubMed: 12371677]

Taylor MJ, Itier RJ, Allison T, Edmonds GE. Direction of gaze effects on early face processing: eyesonly versus full faces. Brain Res Cogn Brain Res. 2001; 10 (3):333-340. [PubMed: 11167057]

Yin RK. Looking at upside-down faces. Journal of Exp Psychol. 1969; 81 (1):141-145. 
Itier et al.

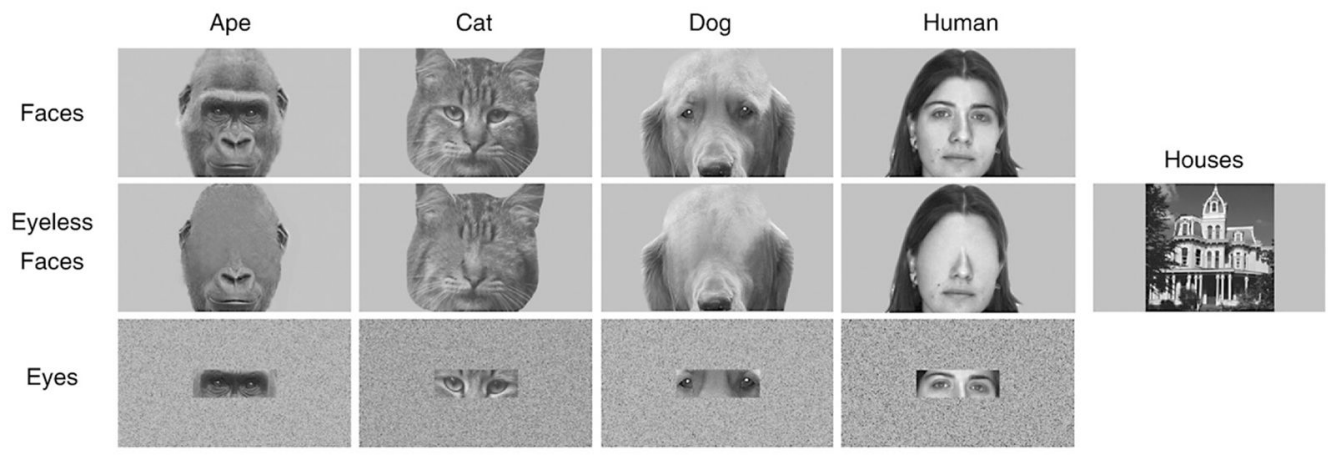

Fig. 1.

Example of stimuli used in the present study. 

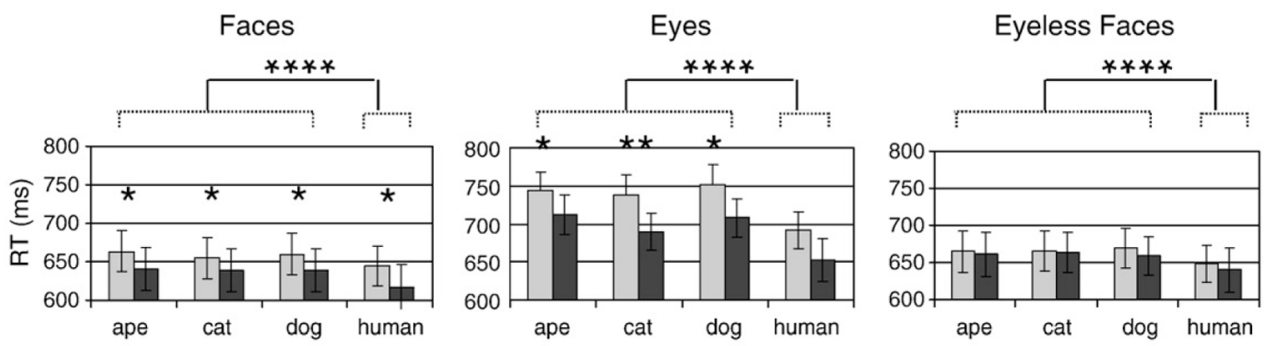

Houses
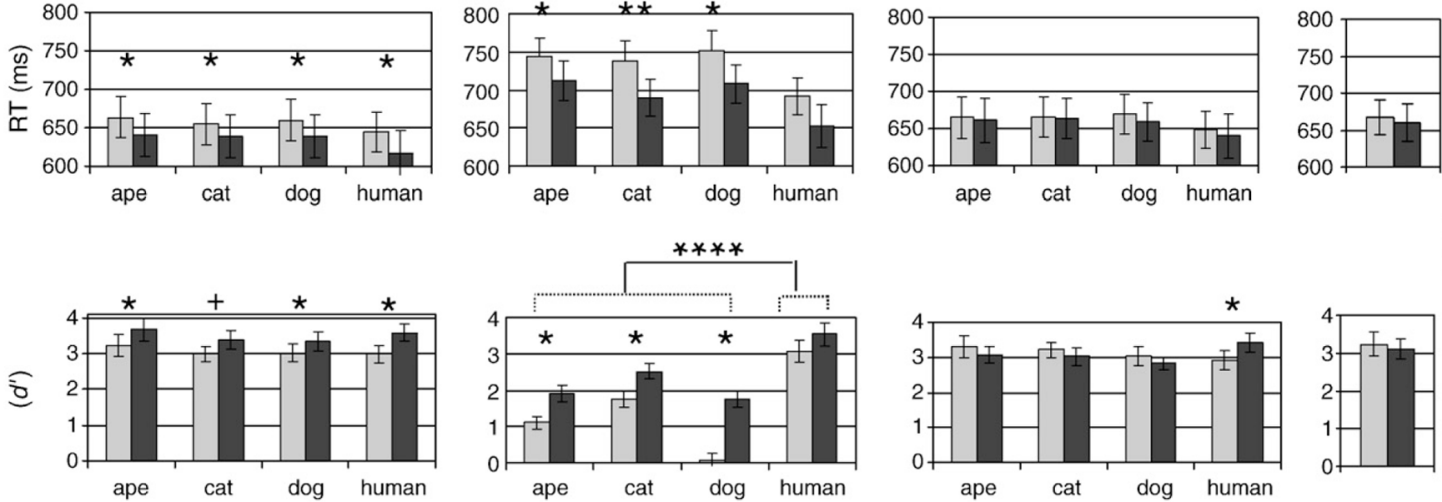

$\square$ inverted

- upright

Fig. 2.

Group mean reaction times (upper panels) and accuracy ( $d^{\prime}$ - lower panels) across species, face conditions, and orientations., + trend $(p=0.07), * p<0.05, * * * * p<0.0001$. 


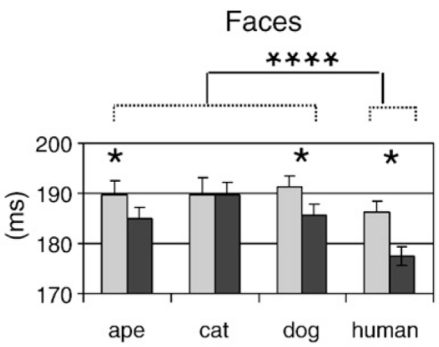

Eyes
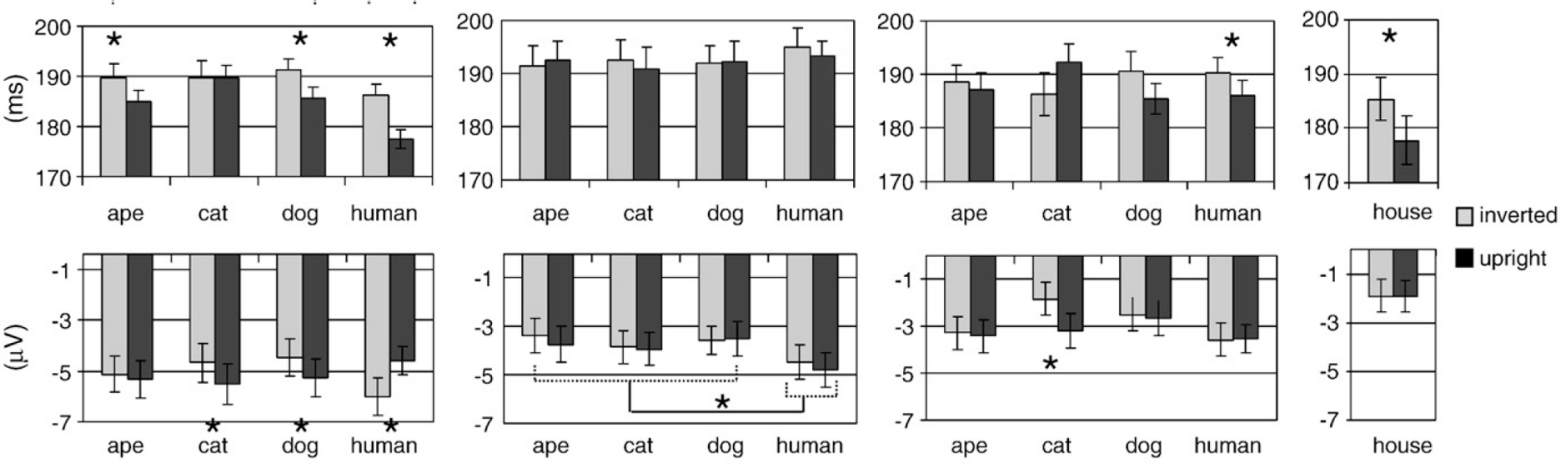

Fig. 3.

Group mean N170 latency (upper panels) and amplitudes (lower panels) across species, face conditions, and orientations (averaged across hemispheres). ${ }^{*} p<0.05$, **** $p<0.0001$. 

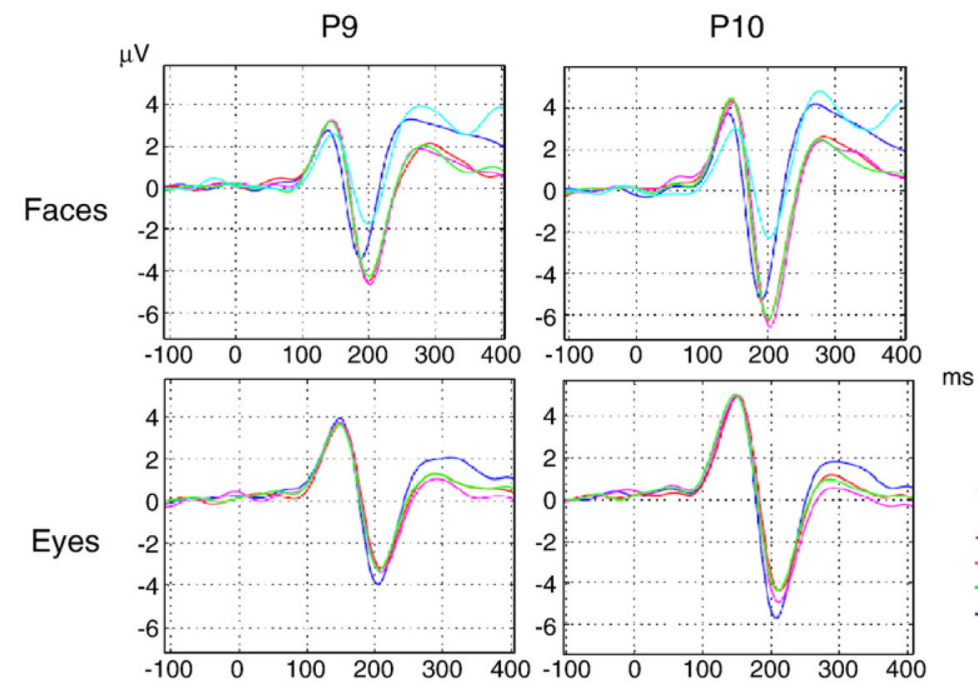

$\mathrm{ms}$

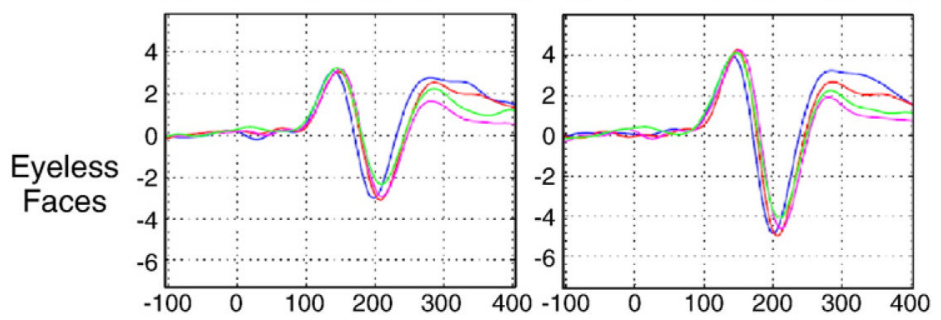

Fig. 4.

Grand averages featuring the N170 modulations for faces (upper panel), eyes (middle panel), and eyeless faces (lower panel) across upright animal categories. The N170 is presented here at P9 and P10 electrodes where amplitudes were largest. 

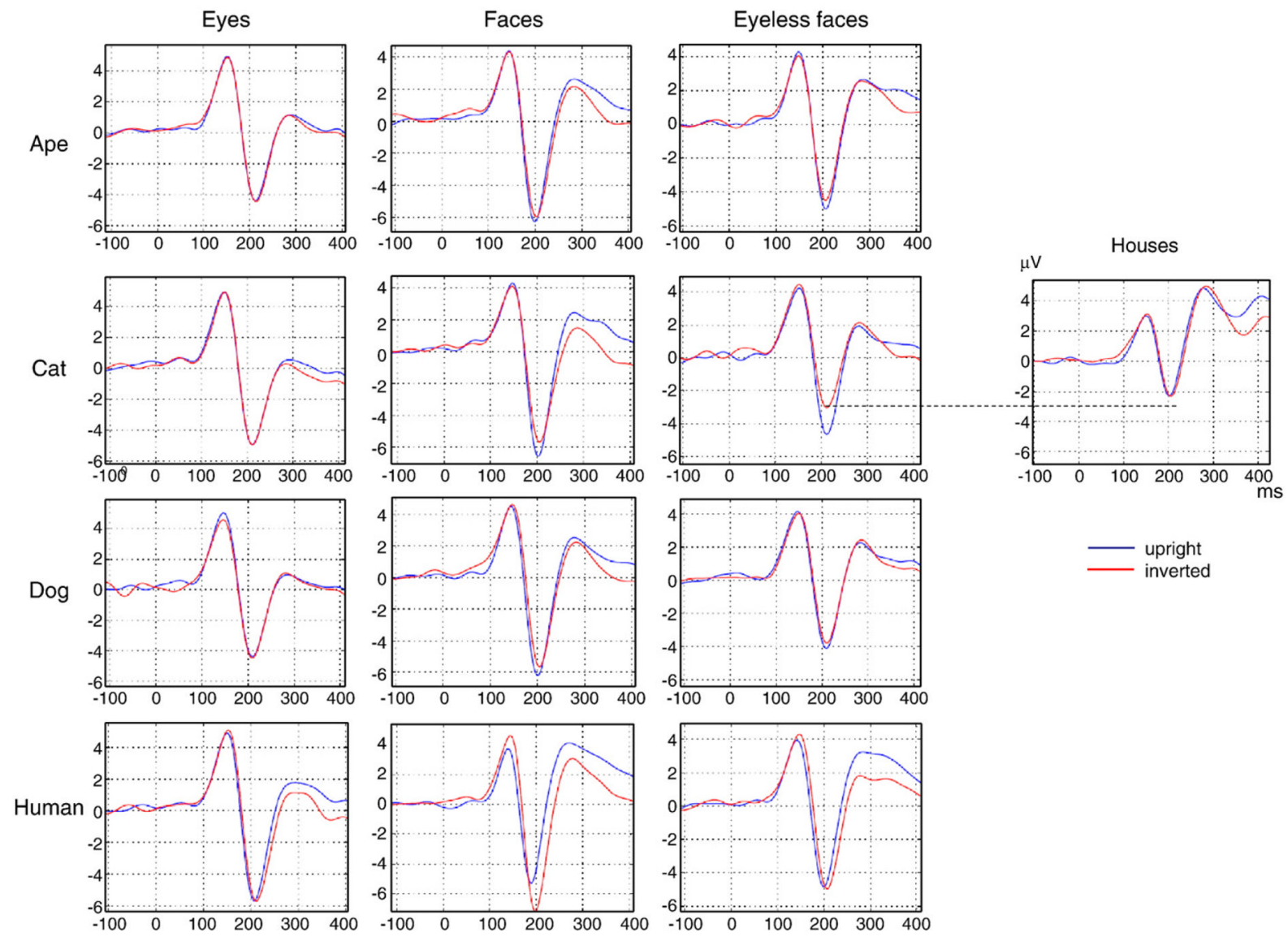

Fig. 5.

Grand averages featuring the N170 modulations with orientation for faces (upper panels), eyes (middle panels), and eyeless faces (lower panels) across animal categories, presented here at P10 electrode. Isolated eyes and eyeless faces did not present inversion effects, except for eyeless cat faces. The normal inversion effect was found for human faces while animal faces did not show modulations with orientation (apes) or showed an inverted inversion effect, i.e. smaller amplitudes for inverted conditions (for cats and dogs). No inversion effect was found for houses. 


\section{Table 1}

Mean luminance $\left(\mathrm{cd} / \mathrm{m}^{2}\right)$ and RMS contrast values across the various species and conditions, standard errors in parentheses.

\begin{tabular}{lcc}
\hline & Mean luminance (std dev) & Mean RMS contrast (std dev) \\
\hline Ape faces & $151.47(11.9)$ & $49.629(9.38)$ \\
Cat faces & $155.8(14.25)$ & $46.86(9.06)$ \\
Dog faces & $153.14(12.5)$ & $52(9.01)$ \\
Human faces & $153.5(19.12)$ & $49.1(12.18)$ \\
Ape eyeless faces & $150.62(12.55)$ & $48.23(10.06)$ \\
Cat eyeless faces & $156.52(14.99)$ & $44.49(10.24)$ \\
Dog eyeless faces & $157.7(12.32)$ & $50.84(9.76)$ \\
Human eyeless faces & $154.61(20.74)$ & $47.51(13.66)$ \\
Ape eyes & $151.45(11.85)$ & $49.66(9.29)$ \\
Cat eyes & $155.94(14.21)$ & $46.73(9.01)$ \\
Dog eyes & $153.21(12.56)$ & $52.01(9.09)$ \\
Human eyes & $153.46(19.14)$ & $49.12(12.16)$ \\
\hline
\end{tabular}

